\title{
SPHERICAL HARMONICS GENERATING BOUNDED BIHARMONICS
}

\author{
BRADLEY BEAVER, LEO SARIO AND CECILIA WANG
}

\begin{abstract}
Let $H^{2} B(R)$ be the family of bounded nonharmonic biharmonic functions on a Riemannian manifold $R$. On the punctured Euclidean $N$-space $E_{0}^{N}=$ $\left\{x=\left(x^{1}, \ldots, x^{N}\right)|0<| x \mid<\infty\right\}, H^{2} B$ is void for $N>3$, whereas for $N=2,3$, it is generated by certain fundamental spherical harmonics. It is also known that $H^{2} B$ remains void on the Riemannian manifold $E_{\alpha}^{N}, N>3$, obtained by endowing $E_{0}^{N}$ with the non-Euclidean metric $d s_{\alpha}=r^{\alpha}|d x|, \alpha \in R$.

The purpose of the present paper is to show that the fundamental spherical harmonics continue generating $H^{2} B\left(E_{\alpha}^{3}\right)$, despite the distorting metric $d s_{\alpha}$. An analogous result holds for $E_{\alpha}^{2}$.
\end{abstract}

Let $H^{2} B(R)$ be the family of bounded nonharmonic biharmonic functions on a Riemannian manifold $R$. It was shown in Sario and Wang [2] that on the punctured Euclidean $N$-space $E_{0}^{N}=\left\{x=\left(x^{1}, \ldots, x^{N}\right)|0<| x \mid<\infty\right\}, H^{2} B$ is void for $N>3$, whereas for $N=2,3$, it is generated by certain fundamental spherical harmonics.

In Sario and Wang [3], it was proved that $H^{2} B$ remains void on the Riemannian manifold $E_{\alpha}^{N}, N>3$, obtained by endowing $E_{0}^{N}$ with the non-Euclidean metric $d s_{\alpha}=r^{\alpha}|d x|, \alpha \in \mathbf{R}$. The cases $N=2,3$ were left open.

The purpose of the present paper is to show that the fundamental spherical harmonics continue generating $H^{2} B\left(E_{\alpha}^{3}\right)$, despite the distorting metric $d s_{\alpha}$. An analogous result holds for $E_{\alpha}^{2}$.

1.1. Consider the space

$$
E_{\alpha}^{N}=\left\{x=\left(r, \theta^{1}, \ldots, \theta^{N-1}\right)\left|0<r<\infty, d s_{\alpha}=r^{\alpha}\right| d x \mid, \alpha \in \mathbf{R}\right\}
$$

with polar coordinates $(r, \theta)=\left(r, \theta^{1}, \ldots, \theta^{N-1}\right)$. We first state some facts from [3].

LEMMA 1. There exist no $H^{2} B$ functions on $E_{-1}^{N}$ for $N \geqslant 2$.

Thus when we are searching for generators of $H^{2} B$ functions, we may ignore the case of $\alpha=-1$.

LEMMA 2. For any $\alpha \neq-1$, and $N \geqslant 2$, every $h \in H\left(E_{\alpha}^{N}\right)$ has an expansion

$$
h=\sum_{n=1}^{\infty} \sum_{m=1}^{m_{n}}\left(a_{n m} r^{p_{n}}+b_{n m} r^{q_{n}}\right) S_{n m}+a \sigma(r)+b,
$$

Received by the editors February 18, 1981.

1980 Mathematics Subject Classification. Primary 31A30, 31 B30.

(1)1982 American Mathematical Society $0002-9939 / 81 / 0000-0794 / \$ 02.50$ 
where

$$
\sigma(r)= \begin{cases}\log r, & \text { if } N=2 \\ r^{-(N-2)(\alpha+1)}, & \text { if } N>2\end{cases}
$$

LEMMA 3. Let $u$ be a biharmonic function on $E_{\alpha}^{N}, N \geqslant 2, \alpha \neq-1$, with

$$
\Delta u=\sum_{n=1}^{\infty} \sum_{m=1}^{m_{n}}\left(a_{n m} r^{p_{n}}+b_{n m} r^{q_{n}}\right) S_{n m}+a \sigma(r)+b .
$$

Then

$$
\begin{aligned}
u= & \frac{-1}{4(\alpha+1)}\left[\sum_{n \neq \mu} \sum_{m=1}^{m_{n}} \frac{1}{P_{n}} a_{n m} r^{p_{n}+2 \alpha+2} S_{n m}+\sum_{n \neq \nu} \sum_{m=1}^{m_{n}} \frac{1}{Q_{n}} b_{n m} r^{q_{n}+2 \alpha+2} S_{n m}\right] \\
& -\frac{1}{2(\alpha+1)} r^{(2-N / 2)(\alpha+1)} \log r \cdot\left(\sum_{m=1}^{m_{\mu}} a_{\mu m} S_{\mu m}+\sum_{m=1}^{m_{\nu}} b_{\nu m} S_{\nu m}\right) \\
& +\sum_{n=1}^{\infty} \sum_{m=1}^{m_{n}}\left(c_{n m} r^{p_{n}}+d_{n m} r^{q_{n}}\right) S_{n m}+a \tau(r)+b s(r)+c \sigma(r)+d,
\end{aligned}
$$

where

$$
\begin{gathered}
p_{n}=\frac{1}{2}\left\{-(N-2)(\alpha+1)+\left[(N-2)^{2}(\alpha+1)^{2}+4 n(n+N-2)\right]^{1 / 2}\right\}, \\
q_{n}=\frac{1}{2}\left\{-(N-2)(\alpha+1)-\left[(N-2)^{2}(\alpha+1)^{2}+4 n(n+N-2)\right]^{1 / 2}\right\}, \\
P_{n}=\frac{1}{2} N(\alpha+1)+p_{n}, \quad Q_{n}=\frac{1}{2} N(\alpha+1)+q_{n},
\end{gathered}
$$

$\mu$ and $\nu$ are defined by $P_{\mu}=0$ and $Q_{\nu}=0$ respectively, $\sigma(r)$ is as above,

$$
s(r)=\frac{-1}{2 N(\alpha+1)^{2}} r^{2 \alpha+2} \text {, }
$$

and

$$
\tau(r)= \begin{cases}s(r)\left[\log r-\frac{1}{\alpha+1}\right], & N=2, \\ -\frac{1}{2 \alpha+2} \log r, & N=4, \\ \frac{1}{2(N-4)(\alpha+1)^{2}} r^{-(N-4)(\alpha+1)}, & N \neq 2,4 .\end{cases}
$$

\subsection{Generators of $H^{2} B$-functions on $E_{\alpha}^{2}$. We claim}

TheOREM 1. $H^{2} B\left(E_{\alpha}^{2}\right)$, where $\alpha=-1 \pm k / 2, k \in Z^{+}$, is generated by $1, \sin k \theta$, and $\cos k \theta$. 
Proof. We shall establish the theorem for the case $\alpha=-1-k / 2$, the other case being entirely similar. Let $S_{n 1}=\cos n \theta$ and $S_{n 2}=\sin n \theta$. From Lemma 3 we calculate

$$
\begin{aligned}
p_{n} & =\frac{1}{2}\left\{-(N-2)(\alpha+1)+\left[(N-2)^{2}(\alpha+1)^{2}+4 n(n+N-2)\right]^{1 / 2}\right\} \\
& =\frac{1}{2}\left[\left(4 n^{2}\right)\right]^{1 / 2}=n, \\
q_{n} & =\frac{1}{2}\left\{-(N-2)(\alpha+1)-\left[(N-2)^{2}(\alpha+1)^{2}+4 n(n+N-2)\right]^{1 / 2}\right\} \\
& =-\frac{1}{2}\left[\left(4 n^{2}\right)\right]^{1 / 2}=-n .
\end{aligned}
$$

$P_{n}=0$ implies $\frac{1}{2} N(\alpha+1)+p_{n}=0$, which yields $n=\frac{k}{2}$. Thus $\mu=\frac{k}{2}$ if $k$ is even. Since $\mu$ must be an integer, there is no $\mu$ term if $k$ is odd.

$Q_{n}=0$ gives $\frac{1}{2} N(\alpha+1)+q_{n}=0$, whence $-n=\frac{k}{2}$, which is impossible, since both $n$ and $k$ are positive. Thus there is no $\nu$ term.

We may represent $u$ by

$$
\begin{aligned}
u= & \sum_{n \neq k / 2} \sum_{m=1}^{2} A_{n m} r^{n-k} S_{n m}+\sum_{n=1}^{\infty} \sum_{m=1}^{2} B_{n m} r^{-n-k} S_{n m} \\
& +r^{-k / 2} \log r \cdot \sum_{m=1}^{2} a_{(k / 2) m} S_{(k / 2) m}+\sum_{n=1}^{\infty} \sum_{m=1}^{2}\left(c_{n m} r^{n}+d_{n m} r^{-n}\right) S_{n m} \\
& +a \tau(r)+b s(r)+c \sigma(r)+d .
\end{aligned}
$$

We wish to show that all coefficients are zero except possibly $A_{k 1}, A_{k 2}$ and $d$.

Suppose $c_{n m} \neq 0$ for some $(n, m)$. Choose $\rho(r) \in C_{0}^{\infty}, \rho \geqslant 0$, supp $\rho \subset(0,1)$. Define $\rho_{t}(r)=\rho(r-t)$ for $0<t<\infty$, and $\varphi_{t}(r, \theta)=\rho_{t} S_{n m}$. Then supp $\varphi_{t} \subset(t, t$ $+1)$, and by the orthogonality of the $\left\{S_{n m}\right\}$,

$$
\begin{aligned}
\left(u, \varphi_{t}\right)= & \int_{E_{\alpha}^{2}} u \rho_{t} S_{n m} r^{-k-1} d \theta d r \\
= & c \int_{t}^{t+1}\left[A_{n m} r^{n-k}+B_{n m} r^{-n-k}\right. \\
& \left.\quad+a_{(k / 2) m} r^{-k / 2} \log r+c_{n m} r^{n}+d_{n m} r^{-n}\right] \rho_{t} r^{-k-1} d r
\end{aligned}
$$

where the third term in the integrand appears only if $n=k / 2$; also,

$$
\left(1,\left|\varphi_{t}\right|\right)=c \int_{t}^{t+1} \rho_{t} r^{-k-1} d r
$$

the $c$ 's denoting constants, not necessarily the same.

Certainly $\varphi_{t} \in L^{1}\left(E_{\alpha}^{2}\right)$, and thus we have the inequality

$$
\left|\left(u, \varphi_{t}\right)\right| \leqslant(\sup |u|)\left(1,\left|\varphi_{t}\right|\right) \text {. }
$$

But as $t \rightarrow \infty,\left(u, \varphi_{t}\right) \sim c t^{n-k-1}$ and $\left(1,\left|\varphi_{t}\right|\right) \sim c t^{-k-1}$, thus forcing $c_{n m}=0$ for all $n$ and $m$. 
If $A_{n m} \neq 0$ for $n>k$, the same estimate with the same test function as in the first step gives, as $t \rightarrow \infty$,

$$
\left(u, \varphi_{t}\right) \sim c t^{n-2 k-1}, \quad\left(1,\left|\varphi_{t}\right|\right)=O\left(t^{-k-1}\right) .
$$

Therefore $A_{n m}=0$ for $n>k$.

Now suppose $B_{n m} \neq 0$ for some $(n, m)$. Choose $\rho(r) \in C_{0}^{\infty}, \rho \geqslant 0$, supp $\rho \subset$ $(\beta, \gamma), 0<\beta<\gamma<1$. Define $\rho_{t}(r)=\rho(r / t)$ for $0<t<1$, and $\varphi_{t}(r, \theta)=\rho_{t} S_{n m}$. Then

$$
\left(u, \varphi_{t}\right)=c \int_{\beta t}^{\gamma t}\left[A_{n m} r^{n-k}+B_{n m} r^{-n-k}+a_{(k / 2) m} r^{-k / 2} \log r+d_{n m} r^{-n}\right] \rho_{t} r^{-k-1} d r,
$$

where the first term in the integrand appears only if $n<k$, and the third only if $n=k / 2$. On the other hand,

$$
\left(1,\left|\varphi_{t}\right|\right)=c \int_{\beta t}^{\gamma t} \rho_{t} r^{-k-1} d r
$$

As $t \rightarrow 0$,

$$
\left(u, \varphi_{t}\right) \sim c t^{-n-2 k}, \quad\left(1,\left|\varphi_{t}\right|\right)=O\left(t^{-k}\right),
$$

and again we have a contradiction to $\varphi_{t} \in L^{1}\left(E_{\alpha}^{2}\right)$. Therefore, $B_{n m}=0$ for all $m$ and $n$.

Suppose $d_{m n} \neq 0$ for some $n \geqslant k$. Using the same test function as above we obtain

$$
\begin{gathered}
\left(u, \varphi_{t}\right)=c \int_{\beta t}^{\gamma t} d_{n m} r^{-n} \rho_{t} r^{-k-1} d r \sim c t^{-n-k}, \quad \text { as } t \rightarrow 0, \\
\left(1,\left|\varphi_{t}\right|\right)=c \int_{\beta t}^{\gamma t} \rho_{t} r^{-k-1} d r=O\left(t^{-k}\right), \quad \text { as } t \rightarrow 0 .
\end{gathered}
$$

A fortiori, $d_{n m}=0$ for $n \geqslant k$.

We have reduced $u$ to

$$
\begin{aligned}
u= & A_{k 1} S_{k 1}+A_{k 2} S_{k 2}+d+\sum_{\substack{n=1 \\
n \neq k / 2}}^{k-1} \sum_{m=1}^{2} A_{n m} r^{n-k} S_{n m} \\
& +r^{-k / 2} \log r \sum_{m=1}^{2} a_{(k / 2) m} S_{(k / 2) m}+\sum_{n=1}^{k-1} \sum_{m=1}^{2} d_{n m} r^{-n} S_{n m} \\
& +a \tau(r)+b s(r)+c \sigma(r) .
\end{aligned}
$$

Since $u$ is bounded, we have, on letting $r \rightarrow \infty, A_{n m}=d_{n m}=0$ for $n=1, \ldots, k-$ 1, and $a_{(k / 2) 1}=a_{(k / 2) 2}=a=b=c=0$. We infer that

$$
u=A_{k 1} S_{k 1}+A_{k 2} S_{k 2}+d=A_{k 1} \cos k \theta+A_{k 2} \sin k \theta+d .
$$

The proof of Theorem 1 is complete.

In the case $k=2$, we have $\alpha=0$, and therefore

Corollary [2]. $H^{2} B\left(E_{0}^{2}\right)$ is generated by $1, \sin 2 \theta$, and $\cos 2 \theta$. 
1.3. Generators of $H^{2} B$ functions on $E_{\alpha}^{3}$. We proceed to show

THEOREM 2. If $\alpha=-1 \pm \sqrt{\frac{1}{2} k(k+1)}, k \in Z^{+}$, then $H^{2} B\left(E_{\alpha}^{3}\right)$ is generated by 1 and $\left\{S_{k m}\right\}_{m=1}^{m_{k}}$, the set of fundamental spherical harmonics of order $k$.

Proof. Again we will give the proof only for $\alpha=-1-\sqrt{\frac{1}{2} k(k+1)}$, the other case being similar. We start with

$$
\begin{aligned}
p_{n} & =\frac{1}{2}\left\{-(3-2)(\alpha+1)+\left[(3-2)^{2}(\alpha+1)^{2}+4 n(n+3-2)\right]^{1 / 2}\right\}, \\
& =\frac{1}{4}\{\sqrt{2 k(k+1)}+\sqrt{2 k(k+1)+16 n(n+1)}\} \\
q_{n} & =\frac{1}{4}\{\sqrt{2 k(k+1)}-\sqrt{2 k(k+1)+16 n(n+1)}\} .
\end{aligned}
$$

If $P_{n}=0$, then $\frac{1}{2} N(\alpha+1)+p_{n}=0$ or $n(n+1)=\frac{3}{8} k(k+1)$. For fixed $k$ this is a quadratic in $n$ which has at most one positive integral solution. If such a solution exists, we denote it by $n=\mu$. Observe that $\mu<k$.

If $Q_{n}=0$, then

$$
-\frac{3}{4} \sqrt{2 k(k+1)}=-\frac{1}{4} \sqrt{2 k(k+1)}+\frac{1}{4} \sqrt{2 k(k+1)+16 n(n+1)},
$$

which has no solution, so that there is no $\nu$ term in $u \in H^{2} B\left(E_{\alpha}^{3}\right)$.

Thus $u$ has the representation

$$
\begin{aligned}
u= & \sum_{n \neq \mu} \sum_{m=1}^{m_{n}} A_{n m} r^{p_{n}+2 \alpha+2} S_{n m}+\sum_{n=1}^{\infty} \sum_{m=1}^{m_{n}} B_{n m} r^{q_{n}+2 \alpha+2} S_{n m} \\
& +r^{(2-N / 2)(\alpha+1)} \log r \cdot \sum_{m=1}^{m_{\mu}} a_{\mu m} S_{\mu m}+\sum_{n=1}^{\infty} \sum_{m=1}^{m_{n}}\left(c_{n m} r^{p_{n}}+d_{n m} r^{q_{n}}\right) S_{n m} \\
& +a \tau(r)+b s(r)+c \sigma(r)+d .
\end{aligned}
$$

The remainder of the proof will be divided into Steps I-V.

I. For any given $n$, the highest order term is obviously $c_{n m} r^{p_{n}}$; we eliminate this first.

Choose $\rho \in C_{0}^{\infty}, \rho \geqslant 0, \operatorname{supp} \rho \subset(0,1)$. Define $\rho_{t}(r)=\rho(r-t)$, and $\varphi_{t}=\rho_{t} S_{n m}$. Then

$$
\begin{gathered}
\left(u, \varphi_{t}\right)=c \int_{t}^{t+1}\left[A_{n m} r^{p_{n}+2 \alpha+2}+B_{n m} r^{q_{n}+2 \alpha+2}\right. \\
\quad+a_{\mu m} r^{\left.-1 / 4(2 k(k+1))^{1 / 2} \log r+c_{n m} r^{p_{n}}+d_{n m} r^{q_{n}}\right]} \\
\quad \cdot \rho_{t} r^{-1-3 / 2(2 k(k+1))^{1 / 2}} d r, \\
\left(1,\left|\varphi_{t}\right|\right)=c \int_{t}^{t+1} \rho_{t} r^{-1-3 / 2(2 k(k+1))^{1 / 2}} d r=O\left(t^{-1-3 / 2(2 k(k+1))^{1 / 2}}\right),
\end{gathered}
$$

whereas

$$
\left(u, \varphi_{t}\right) \sim c t^{p_{n}-1-3 / 2(2 k(k+1))^{1 / 2}}, \text { as } t \rightarrow \infty .
$$

Since $p_{n}>0$ for all $n$, it follows that $c_{n m}=0$ for all $n$ and $m$. 
II. For $n>k, p_{n}+2 \alpha+2>0, p_{n}+2 \alpha+2>q_{n}$, and $p_{n}+2 \alpha+2>\mu$. After Step I above, the latter statements assure us that, for a fixed $n>k, A_{n m} r^{p_{n}+2 \alpha+2}$ is the dominant term as $r \rightarrow \infty$. If $A_{n m} \neq 0$ for $n>k$, the test function from Step I gives

$$
\begin{aligned}
\left(u, \varphi_{t}\right) & =c \int_{t}^{t+1}\left[A_{n m} r^{p_{n}+2 \alpha+2}+\cdots\right] \rho_{t} r^{-1-3 / 2(2 k(k+1))^{1 / 2}} d r \\
& \sim c t^{p_{n}+2 \alpha+2-1-3 / 2(2 k(k+1))^{1 / 2}}, \quad \text { as } t \rightarrow \infty .
\end{aligned}
$$

Thus $A_{n m}=0$ for $n>k$.

III. Suppose $B_{n m} \neq 0$ for some $n$. Choose $\rho \in C_{0}^{\infty}, \rho \geqslant 0$, supp $\rho \subset(\beta, \gamma), 0<\beta$ $<\gamma<1$. Define $\rho_{t}(r)=\rho(r / t), \varphi_{t}=\rho_{t} S_{n m}$. Then

$$
\begin{aligned}
\left(u, \varphi_{t}\right) & =c \int_{\beta t}^{\gamma t}\left[B_{n m} r^{q_{n}+2 \alpha+2}+\cdots\right] \rho_{t} r^{-1-3 / 2(2 k(k+1))^{1 / 2}} d r \\
& \sim c t^{q_{n}+2 \alpha+2-3 / 2(2 k(k+1))^{1 / 2}}, \quad \text { as } t \rightarrow 0, \\
\left(1,\left|\varphi_{t}\right|\right) & =c \int_{\beta t}^{\gamma t} \rho_{t} r^{-1-3 / 2(2 k(k+1))^{1 / 2}} d r=O\left(t^{-3 / 2(2 k(k+1))^{1 / 2}}\right), \quad \text { as } t \rightarrow 0 .
\end{aligned}
$$

The relation $q_{n}+2 \alpha+2<0$ for all $n$ implies $B_{n m}=0$ for all $n$ and $m$.

IV. As in Step II, assume $n>k$. In view of the above, the test function from Step III gives

$$
\left(u, \varphi_{t}\right)=c \int_{\beta t}^{\gamma t} d_{n m} r^{q_{n}} \rho_{t} r^{-1-3 / 2(2 k(k+1))^{1 / 2}} d r \sim c t^{q_{n}-3 / 2(2 k(k+1))^{1 / 2}}
$$

and

$$
\left(1,\left|\varphi_{t}\right|\right)=c \int_{\beta t}^{\gamma t} \rho_{t} r^{-1-3 / 2(2 k(k+1))^{1 / 2}} d r=O\left(t^{-3 / 2(2 k(k+1))^{1 / 2}}\right), \quad \text { as } t \rightarrow 0 .
$$

Inasmuch as $q_{n}<0$ for all $n, d_{n}=0$ for all $n>k$.

$\mathrm{V}$. We have now reduced $u$ to

$$
\begin{aligned}
u= & \sum_{\substack{n=1 \\
n \neq \mu}}^{k} \sum_{m=1}^{m_{n}} A_{n m} r^{p_{n}+2 \alpha+2} S_{n m}+r^{-1 / 4(2 k(k+1))^{1 / 2}} \log r \cdot \sum_{m=1}^{m_{\mu}} a_{\mu m} S_{\mu m} \\
& +\sum_{n=1}^{k} \sum_{m=1}^{m_{n}} d_{n m} r^{q_{n}} S_{n m}+a \tau(r)+b s(r)+c \sigma(r)+d .
\end{aligned}
$$

As in the proof of Theorem 1, we may eliminate a finite number of linearly independent unbounded terms and are left with

$$
u=\sum_{m=1}^{m_{k}} A_{k m} S_{k m}+d
$$

The proof of Theorem 2 is complete.

In particular, when $k=1$, we have $\alpha=0$, and the $S_{1 m}$ are $\cos \theta \cos \psi, \sin \theta \sin \psi$ and $\cos \theta$, where $\theta$ is the angle between the vector and the $z$-axis.

Corollary [2]. $H B^{2}\left(E_{0}^{3}\right)$ is generated by $1, \cos \theta \cos \psi, \sin \theta \sin \psi$, and $\cos \theta$. 


\section{REFERENCES}

1. C. Müller, Spherical harmonics, Lecture Notes in Math., vol. 17, Springer-Verlag, Berlin-HeidelbergNew York, 1966.

2. L. Sario and C. Wang, Generators of the space of bounded biharmonic functions, Math. Z. 127 (1972), 273-280.

3. Math. Soc. (2) 7 (1974), 635-644.

Department of Mathematics, University of California, Los ANgeles, California 90024

Department of Mathematics, Arizona State University, Tempe, Arizona 85281 